\title{
Blended Interaction
}

\author{
Ein neues Interaktionsparadigma
}

\section{Motivation}

Die Arbeitsgruppe Mensch-Computer-Interaktion (MCI) der Universität Konstanz entwickelt neue Konzepte der Interaktion zwischen dem Menschen und dem Computer. Diese basieren auf den neuesten Erkenntnissen der Kognitionsforschung („Embodied Cognition“) und nutzt die aktuellen Entwicklungen der umfassend und allgegenwärtig verfügbaren Computertechnologien (,Ubiquitous Computing“).

Die Grafische Benutzungsoberfläche (Graphical User Interface, kurz GUI) - obwohl schon Ende der 1970er-Jahre in Forschungslaboren entwickelt - stellt immer noch das dominante Interaktionsparadigma dar. Allerdings haben vor allem neuere Erkenntnisse der Kognitionspsychologie maßgeblich dazu beigetragen, dieses Paradigma kritisch zu hinterfragen und weiterzuentwickeln. In der Kognitionspsychologie hat sich in den letzten Jahren eine neue Sichtweise etabliert, die neben den internen, für uns unsichtbaren kognitiven Vorgängen im Gehirn, die Bedeutung unserer Interaktion mit der Umwelt bzw. mit anderen Menschen für die kognitive Entwicklung untersucht (Embodied View of Cognition, siehe dazu [1]). Grundgedanke: Unsere kognitive Entwicklung wird maßgeblich durch unsere körperliche und soziale Interaktion mit Objekten bzw. Lebewesen der Umwelt beeinflusst.

Für die Mensch-Computer Interaktion ergeben sich daraus zumindest zwei Konsequenzen: Anbieten einer reichhaltigen Interaktion, welche möglichst umfassend alle Sinnesorgane sowie unsere körperlichen Fähigkeiten (z. B. Körperbewusstsein, Orientierung im Raum) bei der Benutzung von Computersystemen nutzt. Neben der Sprach- und Stifteingabe spielen hier räumliche Gesten und berührungsempfindliche Displays sowie die Manipulation von Realweltobjekten in Kombination mit digitalen Displays (Tangible Computing) eine wichtige Rolle.

Neben einer reichhaltigen Interaktion soll als weitere Konsequenz die soziale Interaktion (Social Computing) mit anderen Benutzern in die Gestaltung miteinbezogen werden. Dies trägt dem Umstand Rechnung, dass heute vielfach Aufgaben oder Probleme in Gruppen gelöst werden oder Besuche von Museen, Ausstellungen, etc. vielfach in Gruppen stattfinden. Daher ist es wichtig, die sozialen Aspekte der zwischenmenschlichen Kommunikation und Kooperation in die Entwicklung technologischer Anwendungen mit einzubeziehen.

Unter dem Schlagwort Reality-Based Interaction haben Jacob et al. [5] die oben genannten Erkenntnisse der Kognitionspsychologie und die technischen Entwicklungen auf den Gebieten der multimodalen Interaktion $\mathrm{zu}$ einem neuen $\mathrm{Pa}$ radigma zusammengefasst. Ziel ist es dabei, die Interaktion mit dem Computer an der Interaktion mit der realen, nicht digitalen Welt zu orientieren und so realitätsbezogener zu machen.

Daher sind aktuelle Entwicklungen der MenschComputer Interaktion von dem Ziel geprägt, die Interaktion mit einer Vielzahl von unterschiedlichen

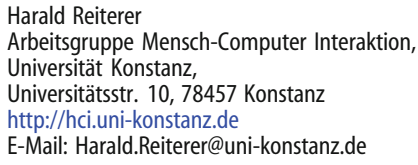




\section{Zusammenfassung}

Dieser Beitrag stellt ein neues Interaktionskonzept namens Blended Interaction zur Gestaltung der Mensch-Computer-Interaktion vor. Es orientiert sich an unseren Erfahrungen der realweltlichen Interaktion mit Objekten und der sozialen Kommunikation und vermischt diese bzw. reichert diese um digitalweltliche Möglichkeiten an.

Endgeräten im Sinne des Ubiquitous Computing [9] an den Prinzipien der Reality-Based Interaction zu orientieren und von den technologischen Möglichkeiten der Augmented Reality [10] umfassenden Gebrauch zu machen. Es kommt dabei zu einer Vermischung von realer und digitaler Welt, daher sprechen wir auch von „Blended Interaction“, wobei aber eine alleinige Steigerung des Realitätsbezuges nicht ausreicht, um eine optimale Interaktion $\mathrm{zu}$ garantieren.

\section{Gestaltungsfelder}

Hier setzt unser neues Interaktionskonzept Blended Interaction an. Es gibt einen methodischen Rahmen vor, bestehend aus Gestaltungsfeldern und einer methodischen Vorgehensweise, wie das Vermischen von Eigenschaften der realen und digitalen Welt erfolgen sollte. Die besondere Herausforderung der Gestaltung und der vom Standpunkt des Benutzers entscheidende Vorteil besteht im Beibehalten erprobter Vorgehensweisen der realen Welt bei gleichzeitiger Anreicherung durch digitale Möglichkeiten. Um diese neue Qualität der Interaktion zu erzielen, müssen zumindest die folgenden vier Bereiche in die Gestaltung der Interaktion miteinbezogen werden.

Individuelle Interaktion: Hier soll eine möglichst intuitive Bedienung erreicht werden, in dem die Benutzer an ihre Alltagserfahrungen hinsichtlich der Interaktion mit Objekten der realen Welt anknüpfen können. Zum Beispiel das Schreiben mit digitalen Stiften auf Papier bewirkt sowohl eine analoge als auch digitale Darstellung; die Interaktion mit technologisch präparierten Realweltobjekten (,Tokens“) vermischt die analoge Interaktion mit dadurch bewirkten digitalen Veränderungen.

Soziale Interaktion und Kommunikation: Hier soll beispielsweise durch „Tokens“ in Kombination mit großformatigen, horizontal oder vertikal angeordneten Multitouch-Displays eine gleichberechtigte Form der Interaktion und Kommunikation ermöglicht werden, da mehrere Benutzer gleichzeitig interagieren bzw. da tradierte soziale Konventionen (z. B. verbale und nonverbale Mechanismen) unmittelbare Berücksichtigung finden können.

Abläufe (Geschäftsprozesse): Hier geht es um die Gestaltung der organisatorischen Einbettung von Abläufen in umfassende Prozesse sowie deren Unterstützung durch Informationstechnologie. Moderne Organisationsstrukturen sind davon gekennzeichnet, dass unterschiedliche Arbeitsprozesse nahtlos aneinander anknüpfen (z. B. Wechsel von Gruppenzu Einzelarbeit, fließender Übergang vom Arbeiten mit realen Objekten wie Papier zu virtuellen Prototypen). Dies gilt nicht nur für die Arbeitswelt, auch Freizeit- oder Ausbildungsaktivitäten sind oft in Abläufen eingebettet: Beispielsweise ist im Rahmen des Besuchs einer Ausstellung ein nahtloser Wechsel zwischen virtuellem und realem Rundgang möglich; beim Recherchieren in einer Bibliothek kann die traditionelle Form der Recherche in Regalen mit den digitalen Möglichkeiten der Recherche kombiniert werden.

Physische Umgebung: Hier ist eine Anpassung der Architektur im weitesten Sinne gemeint: beispielsweise die Anpassung von Möbeln, Räumen, Gebäuden sowie von öffentlichen Plätzen und Städten an neue Formen der technologisch unterstützten Interaktion und Kommunikation. Eine Anpassung erfolgt, indem man die räumlichen Gegebenheiten, aber auch Ton und Licht in die Gestaltung der Interaktion einbezieht und dabei den Formfaktor der Ein- und Ausgabemedien an die räumlichen Gegebenheiten optimal anpasst (z. B. gebogene Displays, touch-sensitive Böden oder Wände).

\section{Methodische Vorgehensweise}

Wir verfolgen den Ansatz, die Theorie und Vorgehensweisen des "Conceptual Blending“ von Fauconnier und Turner [2] auf die Gestaltung der MCI zu übertragen und knüpfen an Arbeiten von Imaz und Benyon [4] an. „Conceptual Blending“ stellt eine Weiterentwicklung der Idee der Nutzung von Metaphern zur Gestaltung der MCI dar. Allerdings vermeidet die Theorie der Blends einige der Nachteile der herkömmlichen Nutzung von Metaphern im Sinne von einfacher 1:1-Übertragung mittels Realweltmetaphern. Typische Beispiele für 


\section{Abstract}

This paper presents a new interaction concept called Blended Interaction for the design of the Human-Computer Interaction. It is based on our experiences interacting with real-world objects and our social communication with people and mixed it and enriches it with the digital power of new computing technologies.

letztere sind die möglichst realistische Nachbildung von Überweisungsformularen, Taschenkalendern oder Taschenrechnern mit allen Beschränkungen, die diese in der realen Welt bieten, auf dem Computer. Ein Blend hingegen weist nicht nur bestimmte partielle Eigenschaften und Strukturen der Quelldomäne und Zieldomäne auf, sondern besitzt auch eigene emergente Strukturen und Eigenschaften. In unserem Beitrag [7] zeigen wir, dass gerade im Bereich der Mensch-Computer-Interaktion Blends sehr hilfreich bei der Entwicklung von mentalen Modellen für den Benutzer, aber auch als Gestaltungsinstrument für den Entwickler sein können. Der theoretisch interessierte Leser sei ausdrücklich auf diesen Beitrag verwiesen, da wir aus Platzgründen eine tiefergehende Erläuterung der theoretischen Grundlagen hier nicht leisten können.

\section{Umsetzung und Erprobung des Konzepts „Blended Interaction“}

Die Umsetzung und Erprobung unseres Interaktionskonzepts „Blended Interaction“ beschränkt sich nicht nur auf die Arbeitswelt (z. B. Design-Meetings oder Einsatzleitsysteme), sondern bezieht auch die Bereiche Freizeit (z. B. Besuch von Ausstellungen und Museen) und Ausbildung (z. B. Recherche in Bibliotheken) mit ein. Dazu wurden im Rahmen von Forschungsprojekten Prototypen entwickelt und erprobt.

\section{Unterstützung von Design-Meetings}

Die Anwendungsdomäne kreativer Gruppenarbeit ist dadurch gekennzeichnet, dass die hier eingesetzten digitalen Werkzeuge den Kreativitätsprozess nicht negativ beeinträchtigen sollten. Papier und Bleistift, Tische und Stellwände, Post-its und Stecknadeln sind immer noch die vorherrschenden Werkzeuge, da alle bisherigen Versuche, geeignete technologische Werkzeuge zu entwickeln, an den pragmatischen Anforderungen der kreativen Praxis gescheitert sind. Daher stellt diese Anwendungsdomäne eine besondere Herausforderung für Blended Interaction Konzepte dar. Mit dem System Affinity Table (siehe Abb. 1) haben wir einen Forschungsprototypen entwickelt, welcher die Vorteile analoger Werkzeuge durch behutsame Anreicherung mit digitaler Funktionalität behält bzw. sogar verstärkt und deren Nachteile zu beseitigen versucht. Das Affinity Table System adressiert alle vier zuvor genannten Designdomänen und Designprinzipien. Die Gestaltung der Individuellen Interaktion wird beispielsweise durch die intuitive Stifteingabe in Kombination mit ,traditionellem“ Papier unterstützt. Die Gestaltung der Sozialen Interaktion wird durch den Multitouch-Tisch in Kombination mit Tokens und digitalen Notizen ermöglicht. Die Gestaltung der physischen Gegebenheiten ergibt sich
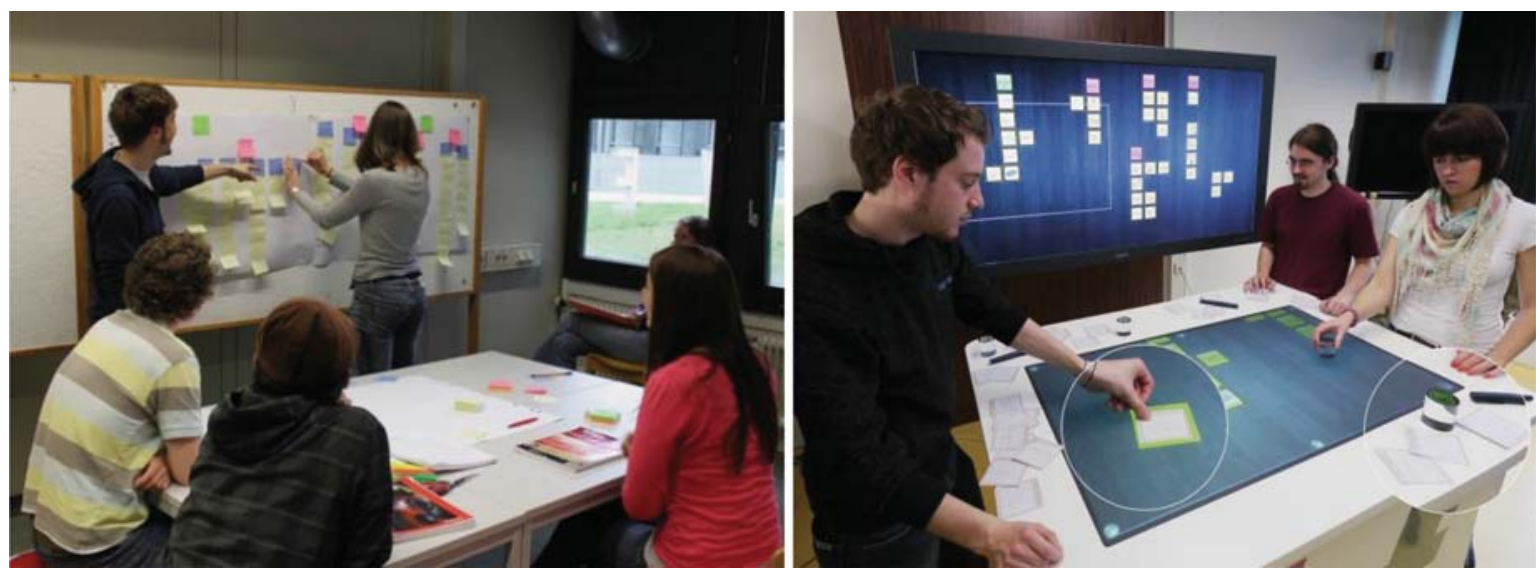

Abb. 1 Herkömmliches Affinity Diagramming (links), der Affinity Table (rechts) 

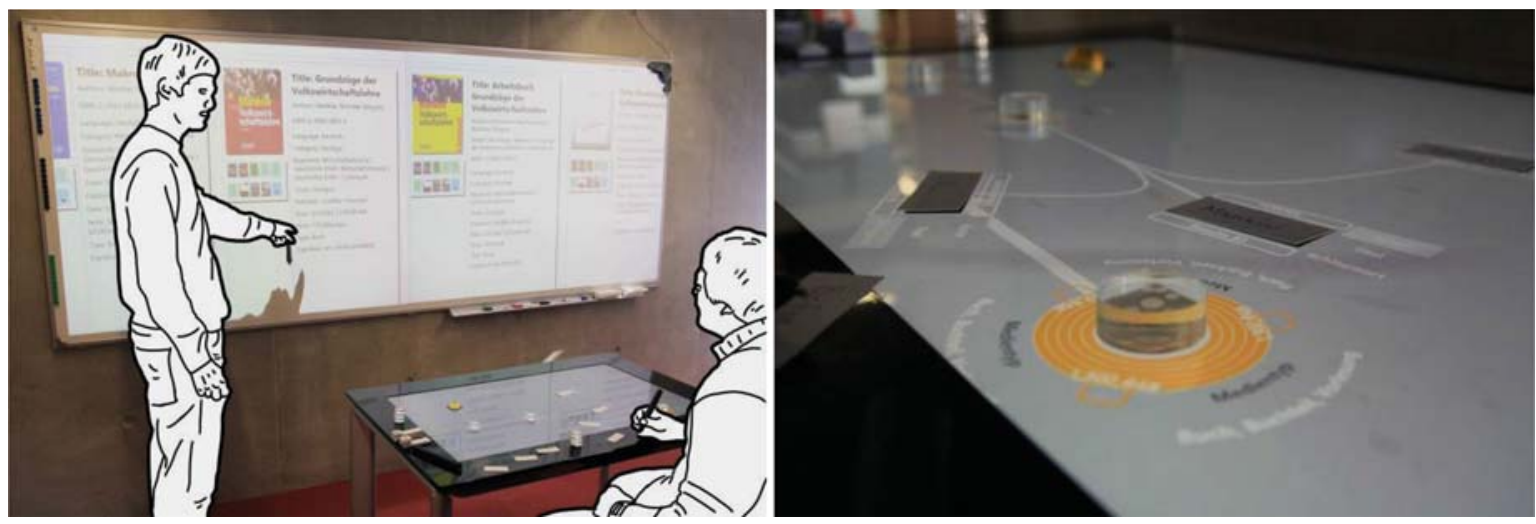

Abb. 2 FacetSearch++ ermöglicht eine kollaborative facettierte Recherche im lokalen Bestand von Bibliotheken. Die facettierte Suche kann mithilfe einer gezielten Stichwortsuche vermischt werden
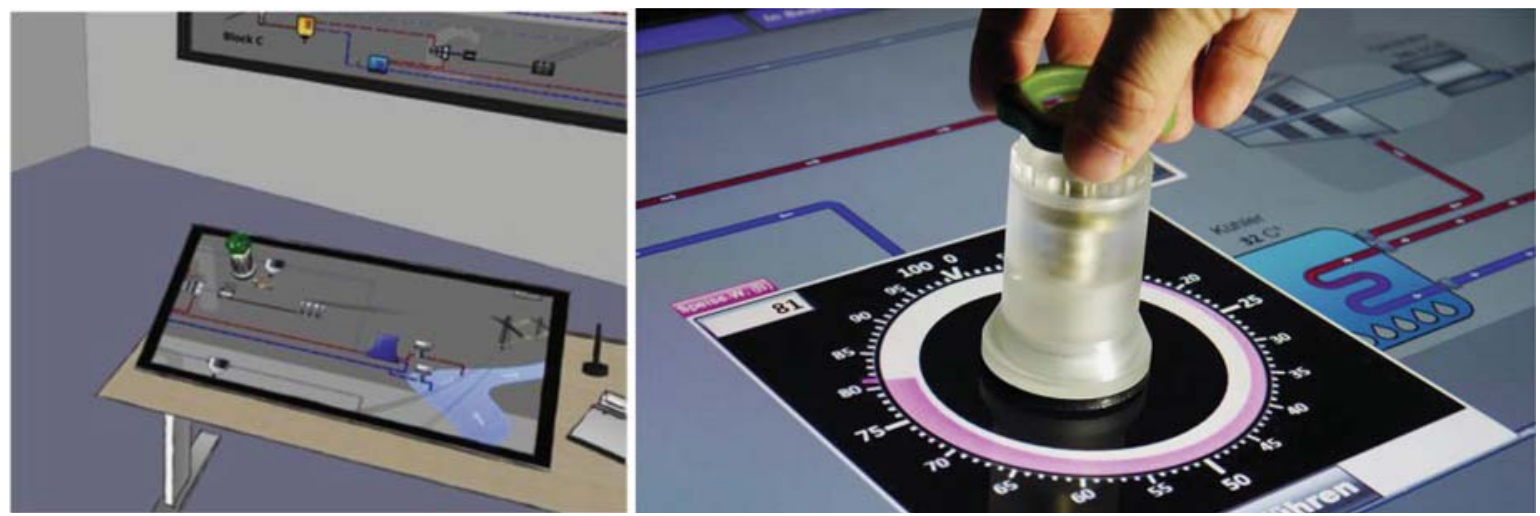

Abb. 3 Darstellung des Prozesses anhand eines digitalen Fließbildes auf einem berührungsempfindlichen Display (links), Manipulation einer Prozessvariable mithilfe eines hybriden Drehreglers (rechts)

durch die gewählte Tisch-Display Kombination. Sie ermöglicht einerseits einen gemeinsamen digitalen Informationsraum sowie individuelle Arbeitsbereiche über den Rahmenbereich des Tisches. Das System unterstützt dabei die Abläufe der Kreativitätstechnik „Affinity Diagramming“, bestehend aus dem individuellen Generieren von Ideen, Diskutieren und Anordnen von Ideen sowie der Bewertung und Selektion von Ideen. Durch die behutsame technologische Erweiterung werden grundlegende Charakteristiken der Methode erhalten und durch sinnvolle Funktionen erweitert (nachzulesen in [3]).

\section{Recherche in Bibliotheken}

Abbildung 2 zeigt Facet Search++, ein Recherchewerkzeug, das eine kollaborative facettierte Suche im lokalen Bestand von Bibliotheken unterstützt. Als Blend dienten hier Brettspiele zur Darstellung des Spielverlaufs, wobei hier die Spielfiguren drehbare Tokens zur Einstellung der Facetten sind. Die visuellen digitalen Verbindungslinien stellen die Ergebnisströme dar. Beide zusammen zeigen den aktuellen Recherchestatus („Spielverlauf“) und auch, welche Facetten („Spielakteure“) für das aktuelle Ergebnis verantwortlich sind (im Detail nachzulesen in [6]). Die facettierte Suche ist um das Konzept der Stichwortsuche erweitert worden und mittlerweile ist eine für die Anfrage an Bibliotheksdaten entwickelte Version in der Universitätsbibliothek Konstanz im Praxiseinsatz.

\section{Überwachung und Manipulation von Prozessen in Einsatzleitsystemen}

Abbildung 3 zeigt einen hybriden Drehregler für die Manipulation numerischer Prozessvariablen von Einsatzleitsystemen (mehr dazu in [8]). Der Regler wird hierbei auf ein digitales Fließbild, welches auf einem berührungsempfindlichen Display dargestellt ist, aufgelegt. Je nach Reglertyp und -positionierung erscheint eine entsprechende Vi- 
sualisierung. Der Blend erschließt sich hierbei aus den haptischen Bedieneigenschaften des physischen Reglers - im Beispiel des Drehreglers der geführten Rotation und der Bedienwiderstände an den Enden des Wertebereiches - und der angepassten digitalen Füllstandanzeige. Somit werden die haptischen Bedieneigenschaften der analogen Manipulation um die vielseitigen Möglichkeiten der digitalen Visualisierung ergänzt.

\section{Literatur}

1. Dourish P (2001) Where The Action Is: The Foundations of Embodied Interaction. MIT Press, Cambridge, MA, USA

2. Fauconnier G, Turner M (2002) The Way We Think: Conceptual Blending and the Mind's Hidden Complexities. Basic Books, New York

3. Geyer F, Pfeil U, Höchtl A, Budzinski J, Reiterer H (2011) Designing Reality-Based Interfaces for Creative Group Work. In: $C \& C^{\prime} 11$ : Proceedings of the 8th ACM Conference on Creativity and Cognition, Atlanta, USA, ACM Press, Honorable
Mention - Best Contribution to Creative Communication Award, November 2011, pp 165-174

4. Imaz M, Benyon D (2007) Designing with Blends: Conceptual Foundations of Human-Computer Interaction and Software Engineering. The MIT Press

5. Jacob RJ, Girouard A, Hirshfield LM, Horn MS, Shaer 0, Solovey ET, Zigelbaum J (2008) Reality-based interaction: a framework for post-WIMP interfaces. In: CHI '08: Proceeding of the Twenty-Sixth Annual SIGCHI Conference on Human Factors in Computing Systems, Florence, Italy, 5-10 April 2008, ACM, New York, NY, pp 201-210

6. Jetter HC, Gerken J, Zöllner M, Reiterer H, Milic-Frayling N (2011) Materializing the Query with Facet-Streams - A Hybrid Surface for Collaborative Search on Tabletops. In: CHI'11: Proceedings of the 29th international conference on Human factors in computing systems, May 2011. ACM Press, CHI 2011 Honorable Mention Paper Award, pp 3013-3022

7. Jetter HC, Reiterer H, Geyer F (2013) Blended Interaction: Understanding Natural Human-Computer Interaction in Post-WIMP Interactive Spaces. Personal and Ubiquitous Computing. Springer, Published online: 18. October 2013

8. Schwarz T, Mueller J, Butscher S, Reiterer H (2012) Holistic Workspace - Neue Interaktionsformen für die Leitwarte der Zukunft. In: Proceedings of Useware 2012, December 2012, VDI

9. Weiser M (1991) The Computer for the Twenty-First Century. Sci Am 09/1991: 94-100

10. Wellner P, Mackay W, Gold R (1993) Computer-Augmented Environments: Back to the real world. Commun ACM 36(7):24-26 